\title{
Immersion Route of Exposure
}

National Cancer Institute

\section{Source}

National Cancer Institute. Immersion Route of Exposure. NCI Thesaurus. Code C85516.

Administration of a medicinal product via partial or complete submersion in a specified

environment such as liquid or air. 